\title{
Ferroelectrically controlled topological magnetic phase in a Janus-magnet-based multiferroic heterostructure
}

\author{
Qirui Cui ${ }^{1,2}$ Yingmei Zhu, ${ }^{1}$ Jiawei Jiang, ${ }^{1}$ Jinghua Liang, ${ }^{1}$ Dongxing Yu, ${ }^{1}$ Ping Cui,${ }^{1,2}$ and Hongxin Yang $\odot^{1, *}$ \\ ${ }^{1}$ Key Laboratory of Magnetic Materials and Devices, Ningbo Institute of Materials Technology and Engineering, \\ Chinese Academy of Sciences, Ningbo 315201, China \\ and Center of Materials Science and Optoelectronics Engineering, University of Chinese Academy of Sciences, Beijing 100049, China \\ ${ }^{2}$ Faculty of Science and Engineering, University of Nottingham Ningbo China, Ningbo 315100, China
}

(Received 14 April 2021; revised 10 August 2021; accepted 10 September 2021; published 5 October 2021)

\begin{abstract}
Electric control of topological magnetic phases has attracted extensive attention due to its potential applications in energy-efficient spintronic devices. Here, using first-principles calculations and atomistic spin model simulations, we demonstrate that electric control of topological magnetic phases can be realized in Janusmagnet-based multiferroic heterostructure, i.e., $\mathrm{MnBi}_{2} \mathrm{Se}_{2} \mathrm{Te}_{2} / \mathrm{In}_{2} \mathrm{Se}_{3}$. The loops of vortices and antivortices can be transformed into skyrmions with diameter of only $4 \mathrm{~nm}$ via ferroelectricity reversal, which is originated from the change of magnetic anisotropy. For heterostructure with up polarization, loops of vortices and antivortices are further tuned to bimeron solitons by applying in-plane magnetic field. Our results thus pave the way for achieving highly tunable topological magnetism in atomic-thickness heterostructure, which can be useful in nonvolatile data encoding and storage with low-energy consumption.
\end{abstract}

DOI: 10.1103/PhysRevResearch.3.043011

\section{INTRODUCTION}

The magnetic skyrmion, as a topologically protected spin texture, is highly promising for nonvolatile information carrier in next-generation spintronic devices with high-storage density and low-energy consumption because it has small size, stable configuration, and can be driven by low-threshold current [1-8]. One key parameter for realizing skyrmion in materials is Dzyaloshinskii-Moriya interaction (DMI), which is originated from spin-orbit coupling (SOC) and inversion symmetry breaking (ISB) [9-12]. In the last decades, many efforts have been devoted to constructing ferromagnetic/heavymetal heterostructures (HSs) with perpendicular magnetic anisotropy (PMA) and large DMI to realize skyrmion [13-17]. Recently, through constructing Janus structures to introduce ISB, the large DMI can be realized in two-dimensional (2D) magnetic materials leading to the formation of skyrmion [18-21]. Compared with multilayers of ferromagnetic/heavy metal, a single 2D magnet has simpler structure, smaller size, and is more beneficial for reducing defects. Besides skyrmion, the bimeron is another topologically nontrivial spin texture consisting of a vortex and an antivortex, which can be constructed by rotating the spin direction of skyrmion by an angle of $90^{\circ}$ [22-24]. The bimeron can also be used as a nonvolatile information carrier for spintronic devices, based on materials with in-plane (IP) magnetic anisotropy (IMA) [25,26].

\footnotetext{
*hongxin.yang@nimte.ac.cn

Published by the American Physical Society under the terms of the Creative Commons Attribution 4.0 International license. Further distribution of this work must maintain attribution to the author(s) and the published article's title, journal citation, and DOI.
}

Another crucial task for practical application of topological spin textures, such as skyrmion and bimeron, is searching or developing a method that can effectively control the mobility and morphology of these spin textures. Particularly, using electric field is considered as a more energy-dissipationless method compared with spin-polarized current, thermal excitation, and external magnetic field $\left(B_{\text {ext }}\right)$ [27-31]. However, since electric field does not break time-reversal symmetry directly, realizing control of magnetism by electricity is difficult [28]. Notably, previous works have demonstrated that the multiferroic system which combines ferroelectricity (FE), ferromagnetism, and ISB provides an ideal platform for electric control of chirality [32,33], creation/annihilation [34], density, and thermal stability [35] of topological magnetism through switching the FE polarization. Here, using first-principles calculations and atomistic spin model simulations, we unveil that a type of FE-controlled topological magnetism can be realized in Janus-magnet-based multiferroic heterostructure, $\mathrm{MnBi}_{2} \mathrm{Se}_{2} \mathrm{Te}_{2}(\mathrm{MBST}) / \mathrm{In}_{2} \mathrm{Se}_{3}$. The loops of vortices and antivortices can be transformed into skyrmions by switching the direction of ferroelectric polarization of $\mathrm{In}_{2} \mathrm{Se}_{3}$. Furthermore, in $\mathrm{MBST} / \mathrm{In}_{2} \mathrm{Se}_{3}$ with up polarization, bimeron solitons can be obtained by applying IP magnetic field. Further analysis unravels that the variation of interlayer charge transfers induced by FE reversal gives rise to the change of magnetic anisotropy, which is responsible for the topological magnetic phase transition in system.

\section{COMPUTATIONAL METHODS}

Our first-principles calculations are performed using the Vienna $A b$ initio Simulation Package (VASP), which is based on the density-functional theory (DFT) [36-38]. The exchange-correlation interaction is described by the 


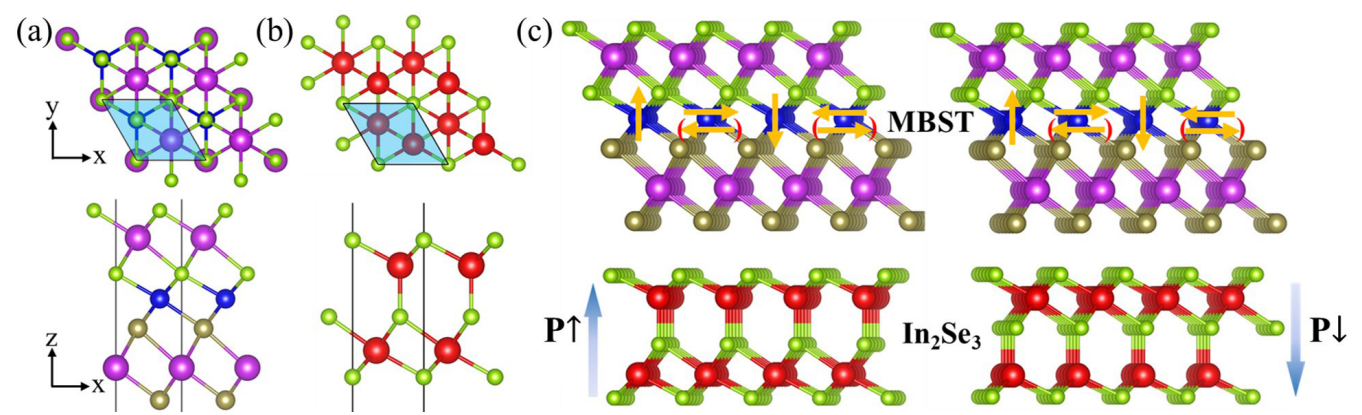

FIG. 1. Top and side views of the crystal structure of (a) MBST and (b) $\operatorname{In}_{2} \mathrm{Se}_{3}$. (c) Side views of MBST/P $\uparrow$ and MBST/P $\downarrow$ HSs. Green, purple, blue, brown, and red balls represent $\mathrm{Se}, \mathrm{Bi}, \mathrm{Mn}, \mathrm{Te}$, and In elements, respectively. The orange vectors in (c) indicate two spin configurations with opposite chirality used to extract the in-plane DMI strength.

generalized gradient approximation (GGA) based on the Perdew-Burke-Ernzerhof function $[39,40]$. The GGA $+U$ method is applied $\left(U_{\text {eff }}=3 \mathrm{eV}\right)$ to describe strongly correlated $3 d$ electrons of Mn [41,42]. The projector-augmented wave method is adopted [43], and the cutoff energy is set to $420 \mathrm{eV}$. The Brillouin zone is sampled using $24 \times 24 \times 1 \Gamma$ center $\boldsymbol{k}$-point grids. The vacuum space of at least $15 \AA$ is used to avoid interactions between periodic replicas. The electronic convergence is performed with a tolerance of $10^{-7} \mathrm{eV}$. All structures are fully relaxed until the Hellmann-Feynman force on each ion is less than $10^{-3} \mathrm{eV} / \AA$. The interlayer van der Waals interaction is corrected by the DFT-D3 method [44]. Dipole corrections are included in calculations of multiferroic HS, and for obtaining the electric polarization of systems, we directly integrate $\rho_{z}$ over the supercell, where $\rho$ represents the local charge density and $z$ represents coordinates along the out-of-plane (OOP) direction [45]. More computational details can be found in the Appendix.

\section{RESULTS AND DISCUSSION}

The crystal structure of Janus MBST monolayer is shown in Fig. 1(a). MBST consists of septuple Te-Bi-Te-Mn-Se-BiSe layers, with atoms in each layer forming a 2D triangular lattice. MBST can be constructed by replacing triple Te-Bi-Te layers of $\mathrm{MnBi}_{2} \mathrm{Te}_{4}$ (MBT) monolayer with triple Se-Bi-Se layers [46], where MBT monolayer is a topologically trivial ferromagnetic semiconductor $[47,48]$. The crystal symmetry of MBST is $C_{3 v}$, and intrinsic ISB allows for DMI between $\mathrm{Mn}$ atoms. The calculated magnetic moment of MBST is $5 \mu_{B}$ per unit cell, indicating that the $\mathrm{Mn}$ atom has a high spin configuration. Furthermore, we demonstrate the dynamic and thermal stability of MBST by calculating the phonon dispersions (see Appendix, 1), elucidating absence of imaginary frequency in Brillouin zone, and ab initio molecular dynamic simulations (AIMD) (see Appendix, 2). Notably, similar ferroelectric heterostructures, such as $\mathrm{WS}_{2} / \mathrm{In}_{2} \mathrm{Se}_{3}$ and $\mathrm{WSe} / \mathrm{In}_{2} \mathrm{Se}_{3}$, have been successfully fabricated in experiments by dry transfer methods [49,50], and Janus monolayers of transition-metal dichalcogenides, i.e., MoSSe and PtSSe, can be synthesized by controlling the reaction atmosphere $[51,52]$. These results highlight the possibility of construction of MBST/In ${ }_{2} \mathrm{Se}_{3} \mathrm{HS}$ in experiments.

$\mathrm{In}_{2} \mathrm{Se}_{3}$ monolayer is a $2 \mathrm{D}$ van der Waals material with room-temperature FE $[45,53]$ and small lattice mismatch with
MBST [54], and its crystal structure is shown in Fig. 1(b). One can see that the $\mathrm{In}_{2} \mathrm{Se}_{3}$ monolayer consists of five triangular atomic layers stacked in sequence of Se-In-Se-In-Se, and the asymmetric position of $\mathrm{Se}$ atoms in the central layer gives rise to the IP and OOP polarizations simultaneously. When $\mathrm{In}_{2} \mathrm{Se}_{3}$ is used as substrate to construct HS with MBST, the reversal of OOP polarization in the $\mathrm{In}_{2} \mathrm{Se}_{3}$ monolayer can induce distinct interfacial coupling, further resulting in the variation of magnetic properties of MBST. Next, we consider 12 possible stacking configurations of $\mathrm{MBST} / \mathrm{In}_{2} \mathrm{Se}_{3} \mathrm{HS}$ as discussed in Appendix, 3. We find that the most stable configuration is the outmost Te and $\mathrm{Bi}$ atoms of MBST sitting at the hollow and top-In sites of $\operatorname{In}_{2} \mathrm{Se}_{3}$, respectively, for both polarized-up $(P \uparrow)$ and -down $(P \downarrow)$ states, and their side views are shown in Fig. 1(c). The equilibrium interlayer distance

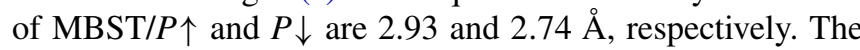
total energy of MBST/P $\uparrow$ is higher by $0.053 \mathrm{eV} /$ u.c. than MBST $/ P \downarrow$, due to the weaker interfacial coupling between MBST and $\operatorname{In}_{2} \mathrm{Se}_{3}$ with upward polarization [55]. The electric

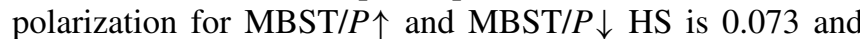
$-0.081 \mathrm{e} \AA /$ u.c., respectively, and the magnitude of that of the $\mathrm{In}_{2} \mathrm{Se}_{3}$ monolayer is $0.096 \mathrm{e} \AA / \mathrm{u}$.c., which implies that the ferroelectricity of $\mathrm{HS}$ mainly comes from $\mathrm{In}_{2} \mathrm{Se}_{3}$. Based on the climbing-image nudged elastic band method, we obtain that the energy barrier of ferroelectric phase transitions of $\mathrm{In}_{2} \mathrm{Se}_{3}$ is $0.083 \mathrm{eV}$. Recent experiments have demonstrated that an OOP field around $1 \mathrm{~V} / \mathrm{nm}$ can overcome such a barrier and flip the OOP polar order [56]. Therefore, it can be expected that a similar electric filed is large enough for realizing the reversal of polar orientation of MBST/In ${ }_{2} \mathrm{Se}_{3} \mathrm{HS}$.

To investigate magnetic properties of systems, we adopt the following spin Hamiltonian:

$$
\begin{aligned}
H= & -J \sum_{\langle i, j\rangle} \boldsymbol{S}_{i} \boldsymbol{S}_{j}-K \sum_{i}\left(S_{i}^{z}\right)^{2}-\sum_{\langle i, j\rangle} \boldsymbol{D}_{i j} \cdot\left(\boldsymbol{S}_{i} \times \boldsymbol{S}_{j}\right) \\
& -\mu_{C r} B \sum_{i} S_{i}^{z}
\end{aligned}
$$

where $S_{i}$ is a unit vector representing local spin of the $i$ th $\mathrm{Mn}$ atom, and $\langle i, j\rangle$ represents summation of all nearestneighboring (NN) Mn pairs. $J, K$, and $\boldsymbol{D}_{i j}$ represent the Heisenberg exchange coupling, magnetic anisotropy, and DMI, respectively. The methods to calculate these parameters are given detail in Appendix, 4, and their results are shown in Table I. We adopt the sign convention that $J>0$ 
TABLE I. Calculated nearest-neighboring exchange coupling $J$, magnetocrystalline anisotropy $K_{\mathrm{MCA}}$, magnetic shape anisotropy $K_{\mathrm{MSA}}$, effective anisotropy $K_{\text {eff }}$, and IP DMI component $d_{\|}$of MBST, $\mathrm{MBST} / P \uparrow$, and MBST $/ P \downarrow$ in units of $\mathrm{meV}$.

\begin{tabular}{|c|c|c|c|c|c|c|c|c|}
\hline & $J_{x x}$ & $J_{y y}$ & $J_{z z}$ & $J$ & $K_{\mathrm{MCA}}$ & $K_{\mathrm{MSA}}$ & $K_{\text {eff }}$ & $d_{\|}$ \\
\hline & & & & & & & & 0 \\
\hline & & & & & 6 & & & 0.3 \\
\hline $\mathrm{BST} / P \downarrow$ & 1.626 & 1.627 & 1.662 & 1.638 & 0.287 & -0.203 & 0.084 & 0.21 \\
\hline
\end{tabular}

$(J<0)$ represents the ferromagnetic (antiferromagnetic) coupling, $K>0(K<0)$ indicates PMA (IMA), and $d_{\|}>0$ $\left(d_{\|}<0\right)$ favors clockwise (anticlockwise) spin configuration. We first calculate the NN exchange coupling for magnetization along positive directions of $x, y$, and $z$ axis, i.e., $J_{x x}, J_{y y}$, and $J_{\mathrm{zz}}$. The SOC effects are taken into account. One can see that the exchange coupling of MBST is ferromagnetic, and $J_{x x}, J_{y y}$, and $J_{z z}$ are almost identical, which indicates that the exchange coupling for MBST is nearly isotropic. Accordingly, we define an isotropic parameter $J$ as the average of $J_{x x}$, $J_{y y}$, and $J_{z z}$, which represents the coefficient in the normally used $-J \sum_{\langle i, j\rangle} S_{i} S_{j}$ term. We also notice that the magnitude of $J$ of MBST is $1.658 \mathrm{meV}$, which is obviously larger than $J=0.922 \mathrm{meV}$ when SOC is not considered. Therefore, the SOC effects cannot be neglected in calculations of exchange coupling for MBST systems. The FE substrate does not change the type of exchange coupling of MBST, and $J$ of $\operatorname{MBST} / P \uparrow(P \downarrow)$ is $1.315(1.638) \mathrm{meV}$. The effective anisotropy $K_{\text {eff }}$ contains two parts: magnetocrystalline anisotropy $K_{\mathrm{MCA}}$ and magnetic shape anisotropy $K_{\mathrm{MSA}}$, where the first part arises from SOC and the second part is caused by magnetostatic dipole-dipole interaction; i.e., $K_{\mathrm{eff}}=K_{\mathrm{MCA}}+K_{\mathrm{MSA}}$. One can see that MBST, MBST $/ P \uparrow$, and MBST/P $\downarrow$ all have positive $K_{\mathrm{MCA}}$. Interestingly, the $K_{\mathrm{MCA}}$ of $\mathrm{HS}$ is significantly enhanced from 0.006 to $0.287 \mathrm{meV}$ when the polarization direction of $\operatorname{In}_{2} \mathrm{Se}_{3}$ is tuned from up to down. Contrary to $K_{\mathrm{MCA}}, K_{\mathrm{MSA}}$ favors the IP alignment of magnetization and basically does not change in three systems. For MBST and $\mathrm{MBST} / P \uparrow, K_{\mathrm{MSA}}$ reaches to -0.205 and $-0.204 \mathrm{meV}$, respectively, whose magnitudes are much larger than $K_{\mathrm{MCA}}$. Therefore, $K_{\text {eff }}$ are negative for both MBST and MBST $/ P \uparrow$. However, due to the giant enhancement of $K_{\mathrm{MCA}}, K_{\mathrm{eff}}$ in MBST/P $\downarrow$ becomes positive. Therefore, the IMA-to-PMA transition is achieved in HS by FE modulation. The transition also indicates that it is very important to investigate $K_{\mathrm{MSA}}$ in calculations of magnetic anisotropy for magnetic thin films. We also test the influence of $U_{\text {eff }}$ on magnetic anisotropy and find that the IMA-to-PMA transition induced by FE reversal is robust to $U_{\text {eff }}$ in the range of 2 to $5 \mathrm{eV}$ (see Table II).

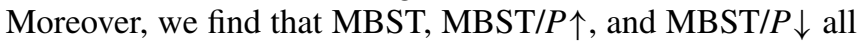
process sizable DMI reaching to $0.307,0.323$, and $0.217 \mathrm{meV}$, respectively. We further calculate the layer-resolved SOC energy difference $\Delta E_{\mathrm{soc}}$ between the opposite chiral spin configurations (see Fig. 2). The detailed computational methods are given in Appendix, 5. For MBST/In ${ }_{2} \mathrm{Se}_{3} \mathrm{HS}$, the DMI still comes from the MBST layer. The Mn layer basically does not contribute to the DMI strength, whereas the most adjacent Te and Bi layers provide dominant contribution, which means
TABLE II. $K_{\mathrm{MCA}}, K_{\mathrm{MSA}}$, and $K_{\text {eff }}$ of HS with $U_{\text {eff }}=2 / 4 / 5 \mathrm{eV}$.

\begin{tabular}{lccccccc}
\hline \hline & \multicolumn{3}{c}{$\mathrm{MBST} / P \uparrow$} & & \multicolumn{3}{c}{$\mathrm{MBST} / P \downarrow$} \\
\cline { 2 - 4 } \cline { 6 - 8 }$U_{\text {eff }}$ & $K_{\mathrm{MCA}}$ & $K_{\mathrm{MSA}}$ & $K_{\text {eff }}$ & & $K_{\mathrm{MCA}}$ & $K_{\mathrm{MSA}}$ & $K_{\text {eff }}$ \\
\hline 2 & 0.008 & -0.198 & -0.190 & & 0.340 & -0.197 & 0.143 \\
4 & 0.015 & -0.209 & -0.194 & & 0.250 & -0.209 & 0.041 \\
5 & 0.024 & -0.214 & -0.190 & 0.230 & -0.214 & 0.016 \\
\hline \hline
\end{tabular}

that the spin tilting is caused by spin-orbit scattering from heavy elements [12].

Once magnetic parameters are obtained, we perform atomistic spin model simulations by using VAMPIRE [57] in square geometry with a side length of $60 \mathrm{~nm}$. In all simulations, the periodic boundary is used along $x$ and $y$ axes, and the initial magnetization state is set to random. More computational details are given in Appendix, 6. The magnetic topology of spin textures can be defined by the topological charge: $Q=\frac{1}{4 \pi} \int \boldsymbol{m} \cdot\left(\partial_{x} \boldsymbol{m} \times \partial_{y} \boldsymbol{m}\right) d x d y$, where $\boldsymbol{m}$ is the normalized magnetization. Both skyrmion and bimeron can be attributed to $Q= \pm 1$. The sign of $Q$ determines the orientation of transverse motion of these topological quasiparticles in the skyrmion Hall effect [3,24]. Figure 3 shows spin textures of MBST, MBST $/ P \uparrow$, and MBST $/ P \downarrow$. For pristine MBST, the loops of vortices and antivortices appear [see Fig. 3(a)], which arise from the competition between DMI and weak IMA [58]. By applying an IP magnetic field along the positive direction of the $x$ axis $(B \rightarrow)$, the vortex-antivortex pairs generate and become ordered along the direction of external field [see Fig. 4(a)]. Interestingly, the bimeron soliton with diameter of around $9 \mathrm{~nm}$ emerges [see Fig. 3(b)] while $B \rightarrow$ increases to $60 \mathrm{mT}$. Compared with MBST, denser loops of vortices and antivortices emerge [see Fig. 3(c)] in MBST/P , since the ferromagnetic exchange coupling is weaker. Moreover, loops in $\mathrm{MBST} / P \uparrow$ can also be transformed into bimeron solitons gradually via enhancing IP magnetic field [see Figs. 3(d) and 4(b)]. Due to the same outer magnetization, bimerons with opposite topological charges $(Q= \pm 1)$ can coexist [23].

The reversal of electric polarization in $\operatorname{In}_{2} \mathrm{Se}_{3}$ from up to down tunes magnetic anisotropy of HS from IP to OOP

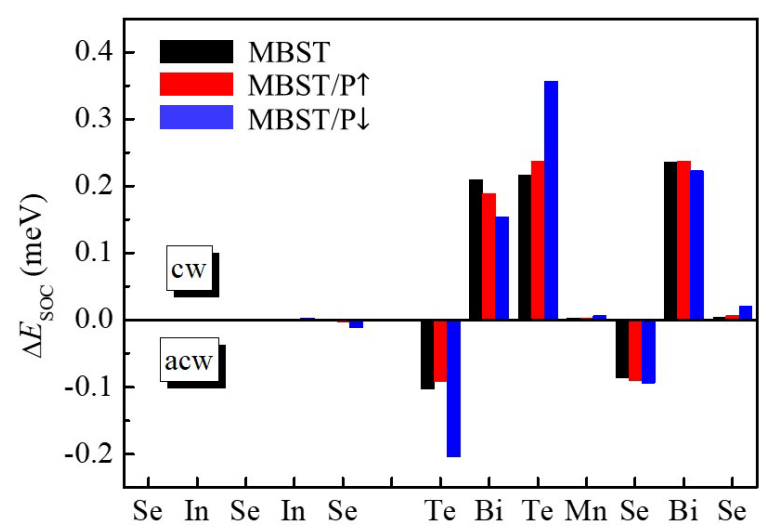

FIG. 2. Layer-resolved localization of the SOC energy difference $\Delta E_{\mathrm{soc}}$ between opposite chiral spin configurations in MBST (black

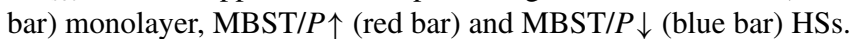


(a) MBST; B $_{\text {ext }}=0 \mathrm{mT}$

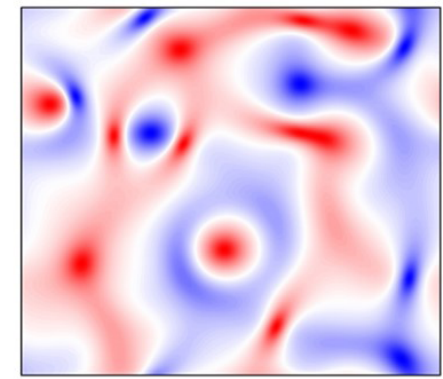

(b) MBST; $\mathrm{B} \rightarrow=60 \mathrm{mT}$

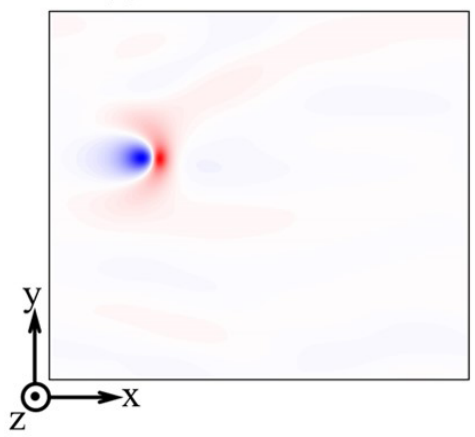

(c) $\mathrm{MBST} / \mathrm{P} \uparrow ; \mathrm{B}_{\text {ext }}=0 \mathrm{mT}$

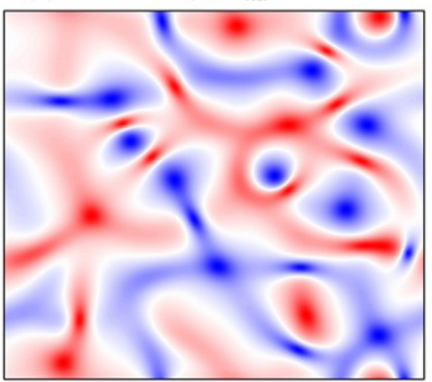

(d) $\mathrm{MBST} / \mathrm{P} \uparrow ; \mathrm{B} \rightarrow=80 \mathrm{mT}$

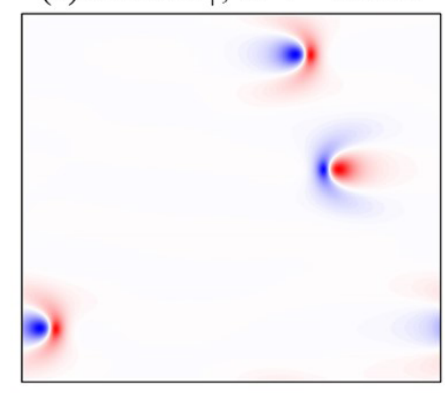

(e) $\mathrm{MBST} / \mathrm{P} \downarrow ; \mathrm{B}_{\text {ext }}=0 \mathrm{mT}$

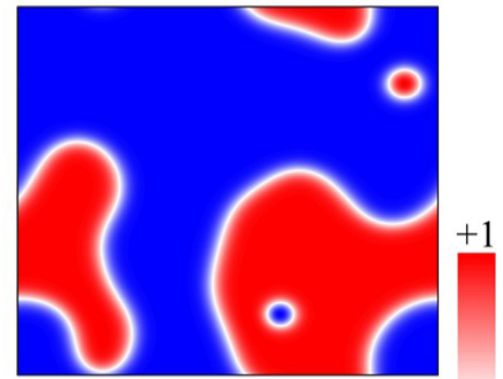

(f) $\mathrm{MBST} / \mathrm{P} \downarrow ; \mathrm{B} \uparrow=80 \mathrm{mT}$

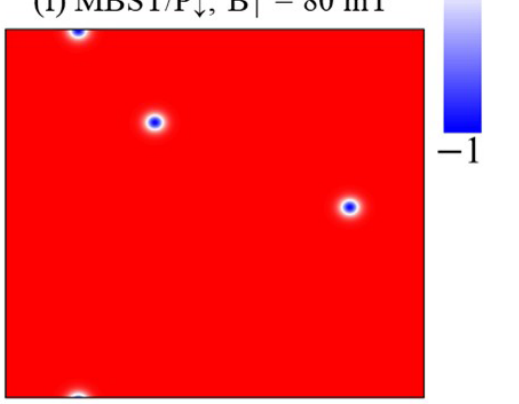

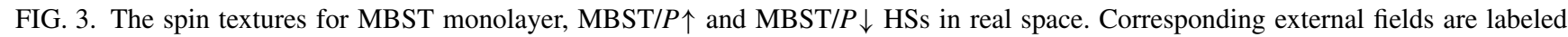
above each panel. The color map indicates the out-of-plane spin component of $\mathrm{Mn}$ atoms.

while the DMI slightly decreases, which indicates that there is obvious variation of topological magnetism. For 2D magnets processing hexagonal lattice, it is convenient to introduce the dimensionless parameter, $\kappa=\left(\frac{4}{\pi}\right)^{2} \frac{2 J K_{\text {eff }}}{3 d_{\|}^{2}}$ [59-61]. The detailed derivation of $\kappa$ is given in Appendix, 7. Notably, $\kappa$ is independent of the magnet thickness but only determined by the magnetic parameters, i.e., $J, K_{\text {eff }}$, and $d_{\|}$, which are directly obtained from first-principles calculations. When $0<$ $\kappa<1$, the magnetic ground state of the system exhibits spin spiral, whereas for $\kappa>1$, the isolated skyrmion could be generated in the background of ferromagnetism. For MBST/P $\downarrow, \kappa$ is 3.15 , which is close to the critical value $(\kappa=1)$ of transition to the spiral state. Therefore, isolated skyrmions are hopefully stabilized. Atomistic spin model simulations demonstrate that the multiple Néel-type skyrmions with diameter of around 4

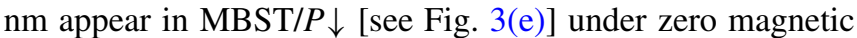
field. We thus obtained the FE-controlled topological mag- (a) MBST; $\mathrm{B} \rightarrow=30 \mathrm{mT}$

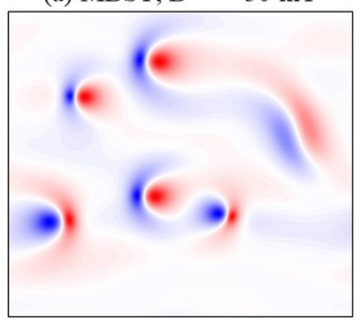

(b) $\mathrm{MBST} / \mathrm{P} \uparrow ; \mathrm{B} \rightarrow=40 \mathrm{mT}$

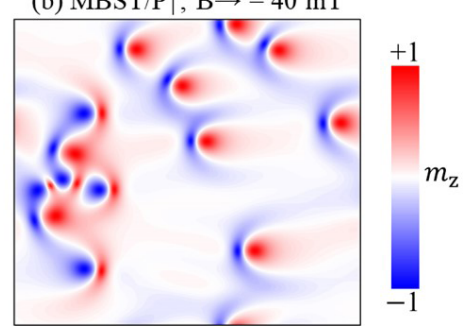

FIG. 4. Spin textures of (a) MBST monolayer under in-plane magnetic field of $30 \mathrm{mT}$ and (b) MBST/P $\uparrow$ HS under IP magnetic field of $40 \mathrm{mT}$. The color map indicates the OOP spin component of Mn atoms. netic structures in MBST/ $\mathrm{In}_{2} \mathrm{Se}_{3}$ HS. The distinct chiral spin textures in MBST $/ P \uparrow$ and MBST/P $\downarrow$ are potentially used as " 0 " and " 1 " bit carriers for realizing the binary data encoding and storage. Strikingly, the size of skyrmion in MBST/P $\downarrow$ is very small, which can largely enhance the integration level of corresponding devices. The skyrmions with opposite topological charges $(Q= \pm 1)$ appear at distinct domains, and applying an $80 \mathrm{mT}$ OOP magnetic field along the positive direction of the $z$ axis ( $\mathrm{B} \uparrow$ ) to shrink the blue domain, the skyrmions with $Q=+1$ are annihilated [see Fig. 3(f)].

Since the FE-controlled magnetic phase transition is dominantly caused by large variation of MCA, we elucidate the physical mechanism of magnetic anisotropy energy (MAE) of $\mathrm{MBST} / \mathrm{In}_{2} \mathrm{Se}_{3} \mathrm{HS}$. From layer-resolved MAE [see Fig. 5(a)], one can find that the MAE of HS just comes from MBST, and the PMA of Te which is adjacent to the Mn atom is enhanced significantly while the polarization of $\operatorname{In}_{2} \mathrm{Se}_{3}$ reverses, which responds for $K_{\mathrm{MCA}}$ increasing. For further understanding MAE change of Te atom, we calculate the MAE from orbital hybridization as shown in Figs. 5(b) and 5(c). One can see that the hybridization between $p_{y}$ and $p_{z}$ provides IMA, while hybridization between $p_{y}$ and $p_{x}$ gives rise to PMA. The PMA contribution arises from $p_{y}$ and $p_{x}$ and is obviously increased when the polarization direction of $\mathrm{In}_{2} \mathrm{Se}_{3}$ reverses from up to down. Moreover, we analyze the MAE change sourcing from $5 p$ orbitals of Te based on second-order perturbation theory [62-64]. MAE can be expressed as

$\mathrm{MAE}=\xi^{2} \sum_{\sigma \sigma^{\prime}} \sum_{\sigma^{\sigma}, u^{\sigma^{\prime}}} \frac{\left(2 \delta_{\sigma \sigma^{\prime}}-1\right)\left(\left|\left\langle o^{\sigma}\left|L_{z}\right| u^{\sigma^{\prime}}\right\rangle\right|^{2}-\left|\left\langle o^{\sigma}\left|L_{x}\right| u^{\sigma^{\prime}}\right\rangle\right|^{2}\right)}{E_{u}^{\sigma^{\prime}}-E_{o}^{\sigma}}$, 

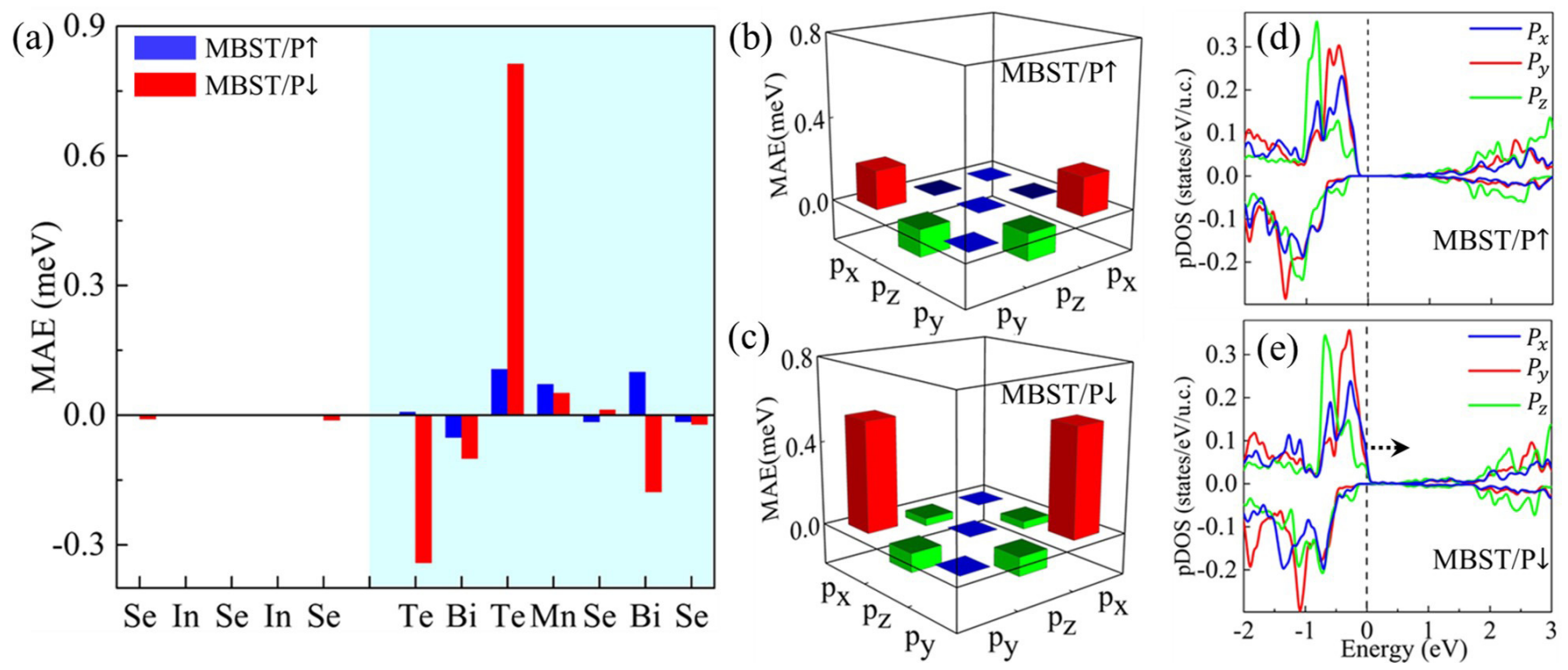

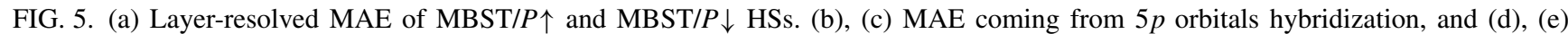
projected density of states of Te which is adjacent to $\mathrm{Mn}$ atom in MBST/In ${ }_{2} \mathrm{Se}_{3} \mathrm{HS}$.

where $\left|o^{\sigma}\right\rangle$ and $\left|u^{\sigma^{\prime}}\right\rangle$ are occupied states with spin $\sigma$ and unoccupied states with spin $\sigma^{\prime}$, respectively, whose energy eigenvalues are $E_{o}^{\sigma}$ and $E_{u}^{\sigma^{\prime}}$. $\left(2 \delta_{\sigma \sigma^{\prime}}-1\right)\left(\left|\left\langle o^{\sigma} L_{z} u^{\sigma^{\prime}}\right\rangle\right|^{2}-\left|\left\langle o^{\sigma} L_{x} u^{\sigma^{\prime}}\right\rangle\right|^{2}\right) \quad$ represents the difference of SOC elements as given in Table III. Figures 5(d) and 5(e) show the projected density of states for Te. One can see that spin-up occupied $p_{x(y)}$ states $\left(p_{x(y)}^{o^{+}}\right)$shift across the Fermi level $\left(E_{F}\right)$ for HS with $P \downarrow$, which is indicated by the dashed black arrow in Fig. 5(e). The difference of SOC elements between $p_{x(y)}^{o^{+}}$and spin-up unoccupied $p_{y(x)}\left(p_{y(x)}^{u^{+}}\right)$is 1 [see Table III], which responds for PMA coming from $p_{x}$ and $p_{y}$ hybridization. The shift of $p_{x(y)}^{o^{+}}$means that the value of $\left(E_{u}^{\sigma^{\prime}}-E_{o}^{\sigma}\right)$ obviously decreases. Therefore, the positive MAE from $p_{x}$ and $p_{y}$ hybridization enhances. It is also found that peaks of spin-up and spin-down occupied $p_{z}$ states $\left(p_{z}^{o^{-}}\right.$and $\left.p_{z}^{o^{+}}\right)$are close to each other. Since SOC elements difference of $p_{y}^{u^{+}}, p_{z}^{o^{-}}$and $p_{y}^{u^{+}}, p_{z}^{o^{+}}$have opposite signs and same amplitudes (see Table III), the MAE contribution from $p_{y}$ and $p_{z}$ barely changes. The fact that FE reversal-induced PMA enhancement is due to the shift of electronic states of Te inspires us to further investigate the interlayer charge transfers of HS. Bader analysis [65-68] shows that the transferred charge from MBST to $\mathrm{In}_{2} \mathrm{Se}_{3}$ obviously increases

TABLE III. The matrix differences between magnetization along positive directions of $x$ and $z$ in Eq. (2). $u^{+}, o^{-}$, and $o^{+}$represent unoccupied spin-up, occupied spin-down and spin-up states, respectively.

\begin{tabular}{rrrrrrrr}
\hline \hline & & \multicolumn{2}{c}{$o^{-}$} & & & \multicolumn{3}{c}{$o^{+}$} \\
\cline { 2 - 4 } \cline { 6 - 8 }$u^{+}$ & $p_{y}$ & $p_{z}$ & $p_{x}$ & & $p_{y}$ & $p_{z}$ & $p_{x}$ \\
\hline$p_{y}$ & 0 & 1 & -1 & & -1 & 1 \\
$p_{z}$ & 1 & 0 & 0 & -1 & 0 & 0 \\
$p_{x}$ & -1 & 0 & 0 & & 0 & 0 \\
\hline \hline
\end{tabular}

from 0.019 to $0.049 \mathrm{e}$ when FE polarization is reversed from up to down, resulting in occupied states of MBST shifting across $E_{F}$ [see Fig. 5(e)]. Furthermore, the planar-average charge-density difference (see Fig. 6) clearly shows that charge transfers are mainly restricted in the interface of $\mathrm{MBST} / P \uparrow$ but spread over all $\mathrm{In}_{2} \mathrm{Se}_{3}$ layer for MBST/P $\downarrow$ [69], which is consistent with charge-transfer analysis.

In summary, using first-principles calculations and atomistic spin model simulations, we demonstrate that by modulating FE polarization of MBST/In ${ }_{2} \mathrm{Se}_{3} \mathrm{HS}$, the IMA-to-PMA transition is achieved, resulting in that loops of vortices and antivortices are transformed into skyrmions with diameter of only $5 \mathrm{~nm}$. Furthermore, the loops in MBST/P $\uparrow$ can be turned to bimeron solitons via applying IP magnetic field. Detailed analysis elucidates that charge transfers between MBST and
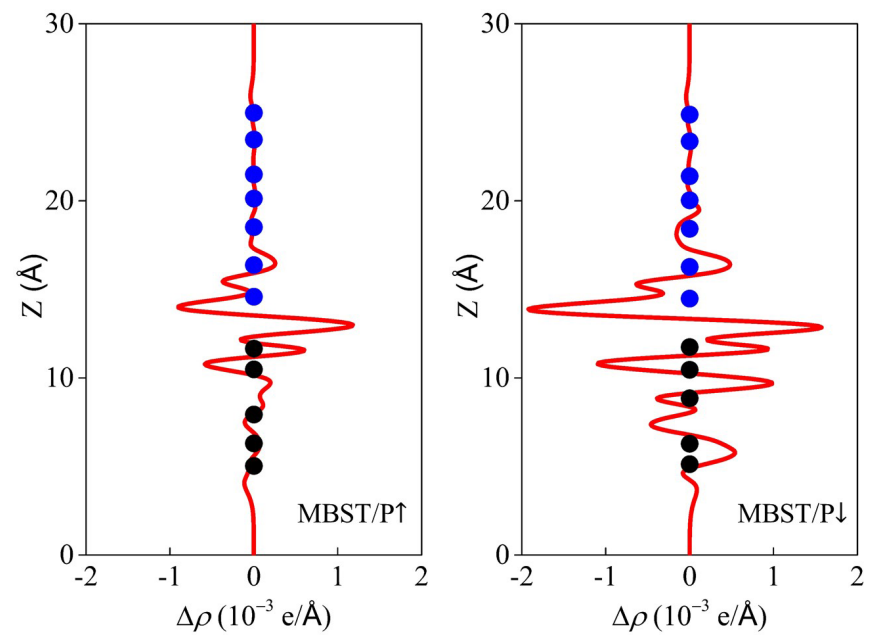

FIG. 6. Planar-average charge-density difference of MBST/P $\uparrow$

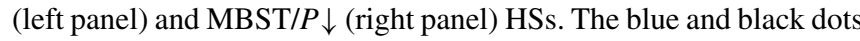
represent the positions of MBST and $\mathrm{In}_{2} \mathrm{Se}_{3}$ atoms, respectively. 


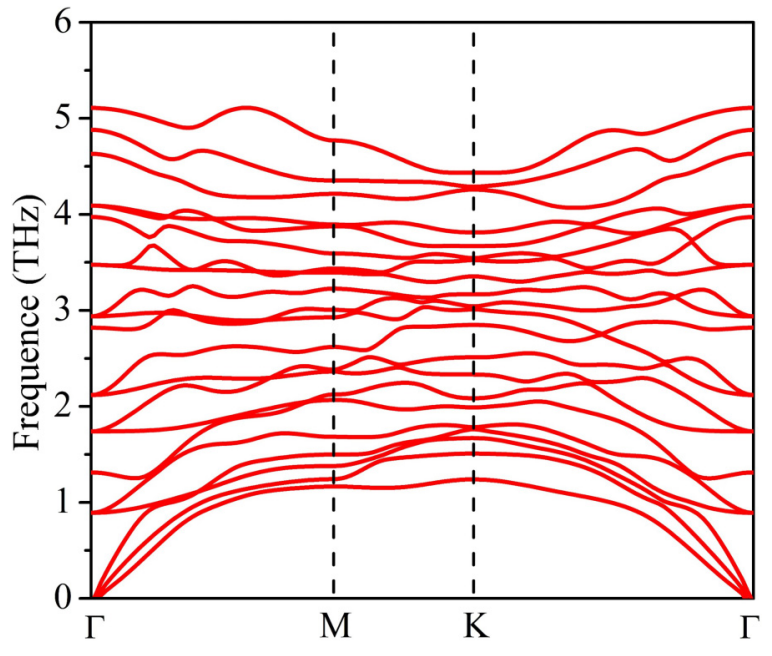

FIG. 7. Calculated phonon band structure for the MBST monolayer.

$\mathrm{In}_{2} \mathrm{Se}_{3}$ play a crucial role in determining magnetic anisotropy of HS. When the FE polarization is reversed from up to down, the $p_{x(y)}^{o^{+}}$states of Te which is adjacent to Mn shifts across the $E_{F}$ due to the increase of interlayer transferred charge, resulting in large enhancement of PMA contribution from Te. The high tunability of topological magnetism is promising to be applied in nonvolatile information handling with lowpower consumption. Since the effect is robust, more nontrivial phenomena of controlling of topological magnetism can be expected in HSs consisting of Janus magnets and ferroelectric/antiferroelectric substrate.

\section{ACKNOWLEDGMENTS}

This work was supported by the National Natural Science Foundation of China, Grants No.11874059 and No. 12174405; Key Research Program of Frontier Sciences, CAS, Grant No. ZDBS-LY-7021; Zhejiang Provincial Natural Science Foundation of China, Grant No. LR19A040002; and Beijing National Laboratory for Condensed Matter Physics.

\section{APPENDIX}

\section{Phonon calculations}

In phonon calculations, the real-space force constants are computed with a $4 \times 4 \times 1$ supercell and $5 \times 5 \times 1 \boldsymbol{k}$ point grids using the VASP code. The force constant matrices and phonon dispersions are calculated using the PHONOPY code [70]. Figure 7 shows the phonon band structure of the monolayer $\mathrm{MnBi}_{2} \mathrm{Se}_{2} \mathrm{Te}_{2}$ (MBST). There is no imaginary frequency, which demonstrates the dynamic stability of MBST.

\section{Molecular dynamics}

For verifying the thermal stability of MBST, we perform AIMD implemented in VASP. The canonical NVT ensemble is used with the Nosé thermostat [71]. For NVT ensemble, the number of particles $(\mathrm{N})$, cell volume $(\mathrm{V})$ and temperature (T) keeps same throughout simulations. A $4 \times 4 \times 1$ supercell with a $1 \times 1 \times 1 \boldsymbol{k}$-point grid is adopted. Each time step is set to $1 \mathrm{fs}$, and simulations are performed for 10000 steps at $300 \mathrm{~K}$. The evolution of the total energy and temperature are shown in Figs. 8(a) and 8(b). The top and side views [see Figs. 8(c) and 8(d)] of MBST after simulations indicate that the structure is still maintained at initial phase.

\section{Heterostructure configurations}

Considering structural symmetries of $\mathrm{In}_{2} \mathrm{Se}_{3}$ and MBST, we propose 12 stacking configurations of $\mathrm{MBST} / \mathrm{In}_{2} \mathrm{Se}_{3} \mathrm{HS}$ (see Fig. 9). The total energies of optimized structures are shown in Fig. 10. Compared with the Se-terminated side, $\mathrm{In}_{2} \mathrm{Se}_{3}$ prefers to contact the Te-terminated side of MBST. For both MBST/P$\uparrow$ and $P \downarrow$, the configuration 6 , with outmost Te and $\mathrm{Bi}$ atoms of MBST sitting at the hollow and top-In sites of $\mathrm{In}_{2} \mathrm{Se}_{3}$, is most stable.

\section{Calculations of $J, K$, and $d_{\|}$}

(i) For obtaining $\mathrm{NN}$ exchange coupling $J$, we compare the energy difference of ferromagnetic and antiferromagnetic states of a $2 \times 1 \times 1$ supercell, i.e., $J=\left(E_{\mathrm{AFM}}-E_{\mathrm{FM}}\right) / 8$. The SOC effects are taken into account. Moreover, to determine the influence of next-nearest-neighboring (NNN) interactions, we expand the unit cell of MBST and calculate the DFT total energies of three different magnetic configurations (see Fig. 11). The total energies of three magnetic configurations are given by

$$
\begin{aligned}
E_{\mathrm{FM}} & =-12 J_{1}-12 J_{2}+E_{\mathrm{other}}, \\
E_{\mathrm{AFM} 1} & =-4 J_{1}+E_{\mathrm{other}}, \\
E_{\mathrm{AFM} 2} & =-4 J_{1}+4 J_{2}+E_{\text {other }},
\end{aligned}
$$

where $J_{1}$ and $J_{2}$ represent $\mathrm{NN}$ and NNN exchange coupling, respectively. Here, SOC effects are also considered, and the magnetization is set to be along the positive direction of the $z$ axis. We find that $J_{1}=1.674 \mathrm{meV}$ and $J_{2}=0.164 \mathrm{meV}$. The magnitude of $J_{1}$ is much larger than that of $J_{2}$, and is very close to the that directly obtained from the $2 \times 1$ supercell. These results indicate that the NNN coupling is almost negligible in MBST systems. (ii) The magnetocrystalline anisotropy $K_{\mathrm{MCA}}$ is calculated as: $K_{\mathrm{MCA}}=E_{M \rightarrow}-$ $E_{M \uparrow}$, where $E_{M \rightarrow}$ and $E_{M \uparrow}$ represent the self-consistent total energy of system with magnetization along the positive direction of $x$ and $z$ axes, respectively, when SOC effect is included. The magnetic shape anisotropy $K_{\mathrm{MSA}}$ is the sum of all magnetostatic dipole-dipole interactions up to infinity $[72,73]$ and is calculated by $K_{\mathrm{MSA}}=E^{\text {dip }} / V_{\text {u.c. }}=$ $-\frac{1}{2} \frac{\mu_{0}}{4 \pi V_{\text {u.c. }}} \sum_{i \neq j}^{N} \frac{1}{r_{i j}{ }^{3}}\left[\boldsymbol{M}_{i} \cdot \boldsymbol{M}_{j}-\left(\boldsymbol{M}_{i} \cdot \boldsymbol{r}_{i j}\right)\left(\boldsymbol{M}_{j} \cdot \boldsymbol{r}_{i j}\right) \frac{3}{r_{i j}{ }^{2}}\right]$, where $\boldsymbol{M}_{i}$ and $\boldsymbol{r}_{i j}$ represent local magnetic moments and vector connecting site $i$ and $j$, respectively. A $250 \times 250 \times 1$ supercell is used in $K_{\mathrm{MSA}}$ calculation to ensure the reliability of results. Also, we would like to note that skyrmions and merons are small topological defects on the uniform magnetic background $[4,6]$, which indicates that shape anisotropy can be considered as the contribution to effective magnetic anisotropy. This approximation has been used in micromagnetic simulations of complex spin textures, such as skyrmion and chiral domain wall [1,32], and gives good agreement with experiments [13]. (iii) The chirality-dependent total energy 

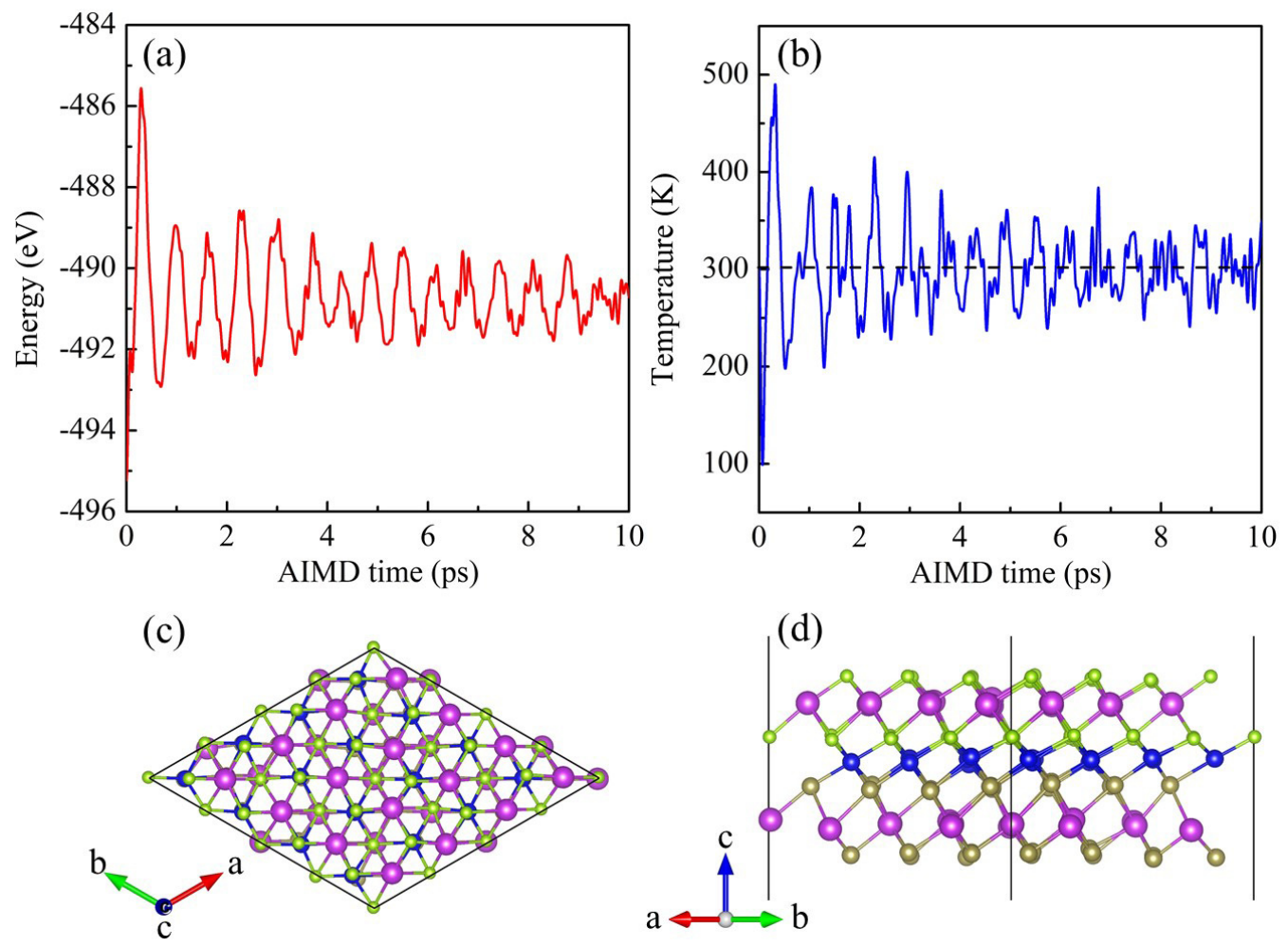

FIG. 8. (a) The evolution of total energy and (b) temperature during the ab initio simulations for MBST monolayer at $300 \mathrm{~K}$. The (c) top and (d) side views of MBST supercell after simulations of $10 \mathrm{ps}$.

difference approach is applied to obtain the DMI strength [12]. Since the out-of-plane components cancel each other, we only provide the in-plane component of DMI, $d_{\|}$. In calculations of DMI, a $4 \times 1 \times 1$ supercell with $6 \times 24 \times 1 \boldsymbol{k}$-point grids is

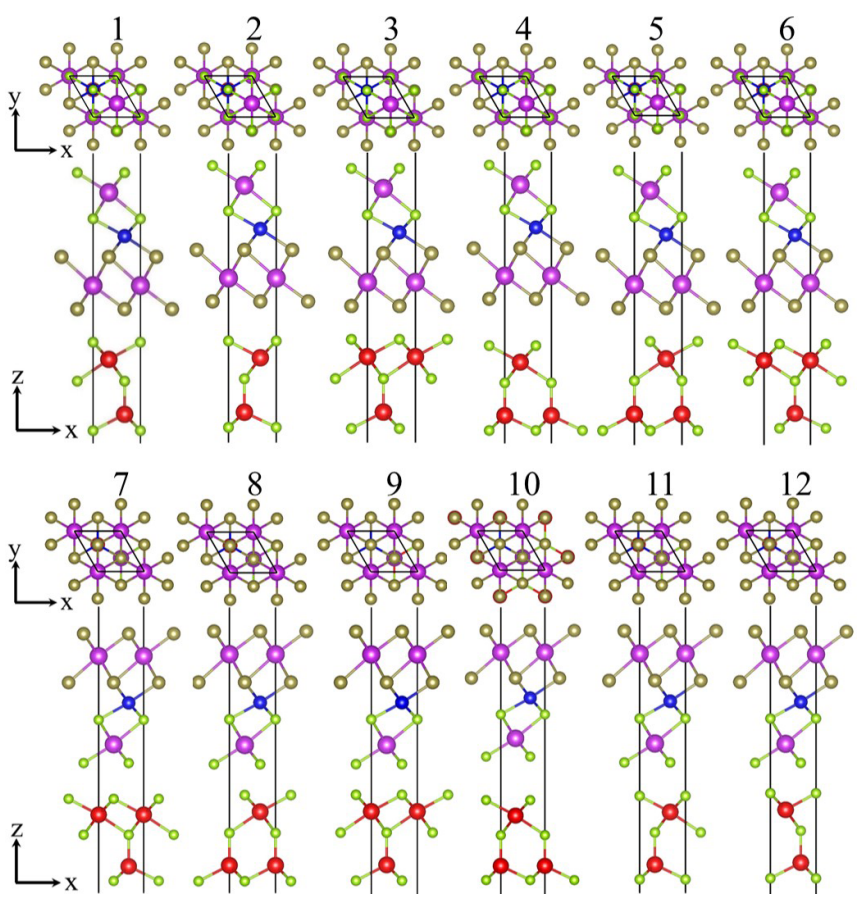

FIG. 9. Top and side views of 12 stacking configurations for $\mathrm{MBST} / \mathrm{In}_{2} \mathrm{Se}_{3} \mathrm{HS} \mathrm{s}$. adopted. To obtain $d_{\|}$, we calculate the energies of clockwise $(\mathrm{CW})$ and anticlockwise (ACW) spin configurations as shown in Fig. 1(c) of the main text, and $d_{\|}$equals $\left(E_{\mathrm{ACW}}-E_{\mathrm{CW}}\right) / 12$. (iv) In fact, the spin Hamiltonian for describing spin interactions between Mn atoms can be written as $H=\boldsymbol{S}_{i} \cdot \overleftrightarrow{\boldsymbol{J}} \cdot \boldsymbol{S}_{j}$, where the exchange matrix has components

$$
\overleftrightarrow{J}=\left[\begin{array}{ccc}
J_{x x} & \Gamma_{x y}+D_{z} & \Gamma_{x z}-D_{y} \\
\Gamma_{x y}-D_{z} & J_{y y} & \Gamma_{y z}+D_{x} \\
\Gamma_{x z}+D_{y} & \Gamma_{y z}-D_{x} & J_{z z}
\end{array}\right] .
$$

Here, the $J$ and $D$ represent Heisenberg exchange coupling and DMI, respectively. The $\Gamma_{x y}, \Gamma_{x z}$, and $\Gamma_{y z}$ are the additional, symmetric, off-diagonal contributions to the exchange tensor. For determining $\Gamma$ of MBST, such as $\Gamma_{x y}$, we compare the energies of a $2 \times 1 \times 1$ supercell with spin configurations

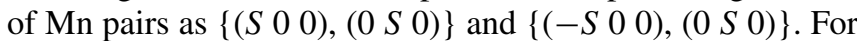

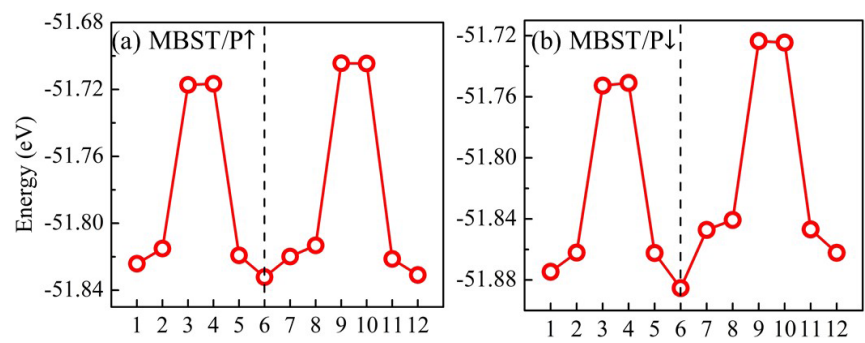

FIG. 10. Total energies of 12 stacking configurations for (a) MBST/P $\uparrow$ and (b) MBST $/ P \downarrow$ HSs. The dashed line indicates the most stable stacking. 


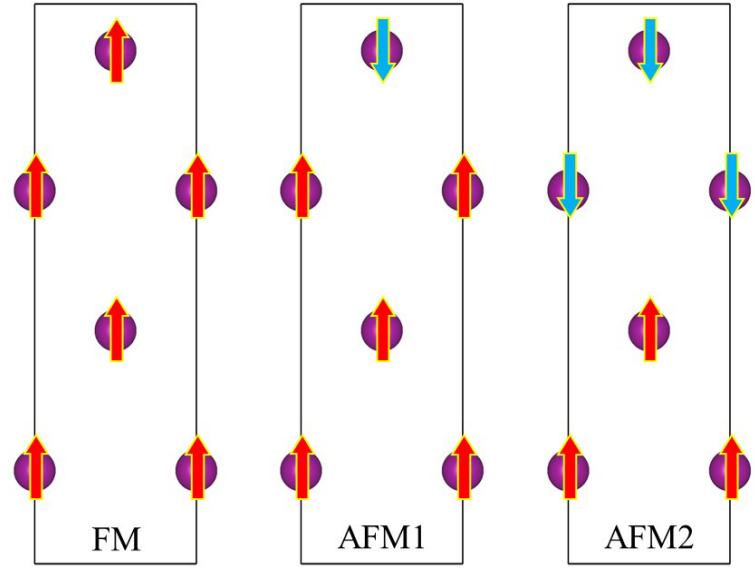

FIG. 11. Three different spin configurations applied to calculate the $\mathrm{NN}$ and $\mathrm{NNN}$ exchange coupling parameters.

$\left\{\left(\begin{array}{lll}S & 0 & 0)\end{array}\right),(0 S 0)\right\}, E_{1}=J_{x x}+J_{x y}+4 \Gamma_{x y}$, and for $\left\{\left(\begin{array}{lll}S & 0 & 0\end{array}\right),(0 S\right.$ $0)\}, E_{2}=J_{x x}+J_{x y}-4 \Gamma_{x y}$. Accordingly, $\Gamma_{x y}=\left(E_{1}-E_{2}\right) / 8$. $\Gamma_{x y}, \Gamma_{x z}$, and $\Gamma_{y z}$ of MBST are $0.0027,0.0038$, and 0.0018 $\mathrm{meV}$, respectively. One can see that the magnitude of $\Gamma$ is very limited and is much smaller compared with DMI components. These results indicate that $\Gamma$ can be negligible for MBST systems.

\section{Calculations of layer-resolved localization of the SOC energy difference $\Delta E_{\text {soc }}$ between opposite chiral spin configurations and layer-resolved MAE}

The SOC is calculated for each atom layer in MBST and $\mathrm{MBST} / \mathrm{In}_{2} \mathrm{Se}_{3} \mathrm{HSs}$. By comparing the atom-layer SOC energy between ACW and CW configurations, and in-plane (100) and out-of-plane (001) magnetic orientations, one can obtain layer-resolved $\Delta E_{\mathrm{soc}}$ and MAE, respectively.

\section{Atomic spin model simulations}

All atomic spin model simulations are performed using the VAMPIRE package [57]. The hexagonal lattice of MBST is transformed into a lattice with an orthorhombic unit cell (see Fig. 12). Then, this unit cell is extended to a $60 \times 60-\mathrm{nm}$ square with the periodic boundaries.

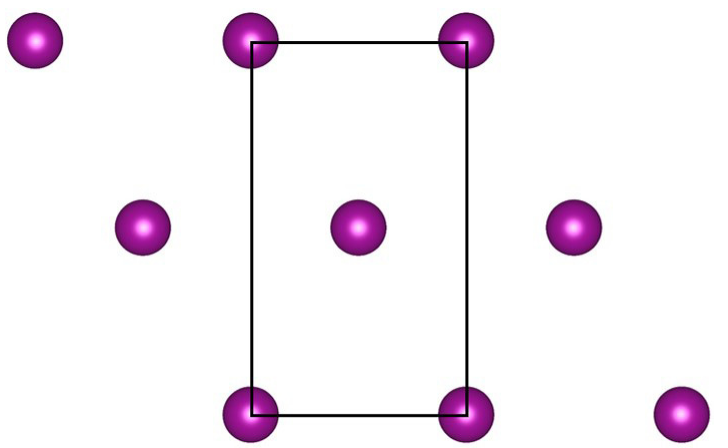

FIG. 12. The orthorhombic unit cell for MBST and $\mathrm{MBST} / \mathrm{In}_{2} \mathrm{Se}_{3}$ HSs. The purple atoms represent Mn element.
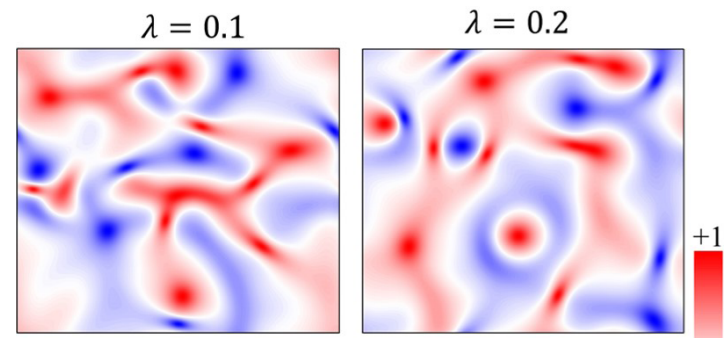

$\lambda=0.3$
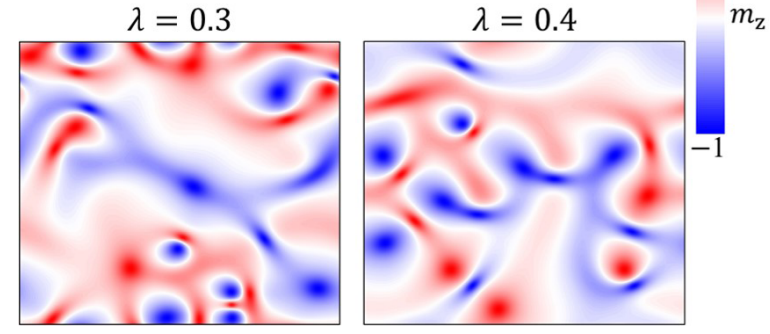

FIG. 13. The spin textures for MBST monolayer with $\lambda$ in range of 0.1 to 0.4 . The color map indicates the out-of-plane spin component of Mn atoms.

The time evolution of atomic spin is described by the Landau-Lifshitz-Gilbert (LLG) equation. The LLG is given by: $\frac{\partial \boldsymbol{S}_{i}}{\partial t}=-\frac{\gamma}{\left(1+\lambda^{2}\right)}\left[\boldsymbol{S}_{i} \times \boldsymbol{B}_{\text {eff }}^{i}+\lambda \boldsymbol{S}_{i} \times\left(\boldsymbol{S}_{i} \times \boldsymbol{B}_{\text {eff }}^{i}\right)\right]$, where $\boldsymbol{S}_{i}$ is the unit vector of the spin moment of site $i, \gamma$ is gyromagnetic ratio, and $\lambda$ represents damping constant. $\boldsymbol{B}_{\text {eff }}^{i}$ represents the effective magnetic field on each spin and can be expressed as $\boldsymbol{B}_{\text {eff }}^{i}=-\frac{1}{\mu_{s}} \frac{\partial H}{\partial S_{i}}$, where $H$ is the spin Hamiltonian of MBST. We choose $\lambda=0.2$ for MBST, considering magnetism originates from $3 d$ electrons of $\mathrm{Mn}$, and $\mathrm{Bi}$ and Te process strong SOC [74]. Moreover, we calculate spin textures of MBST with $\lambda$ in the range of 0.1 to 0.4 (see Fig. 13). One can find that loops of vortices and antivortices emerge, which are robust to the value of $\lambda$.

\section{The $\kappa$ for MBST monolayer}

The dimensionless parameter $\kappa$ is calculated by $\kappa=$ $\left(\frac{4}{\pi}\right)^{2} \frac{A K}{D^{2}}$, where $A$ is exchange stiffness, and $K$ and $D$ are the magnetic anisotropy and DMI parameter in micromagnetic format, respectively. Based on the Heisenberg model, the exchange energy can be expressed as $E_{\mathrm{ex}}=J \sum_{i, \delta}\left(\boldsymbol{\delta}_{i} \cdot \nabla \boldsymbol{m}_{i}\right)^{2}$, where $J$ is the magnitude of exchange coupling, and $\delta_{i}$ is the vector between neighboring local spin $\boldsymbol{m}_{i}$. In micromagnetic format, $E_{\mathrm{ex}}=A \int d V\left[\left(\nabla m_{x}\right)^{2}+\left(\nabla m_{y}\right)^{2}+\left(\nabla m_{z}\right)^{2}\right]$, where $A$ is the exchange stiffness. For MBST monolayer, one can obtain $A=\frac{\sqrt{3} J}{t}$, where $t_{F}$ is the assumed thickness of magnetic film. The micromagnetic anisotropy is calculated as $K=\frac{K_{\text {eff }}}{V}=\frac{2 K_{\text {eff }}}{\sqrt{3} a^{2} t_{F}}$, where $a$ is the lattice constant. The micromagnetic DMI strength $D$ is calculated as $D=\frac{d_{\|} \sqrt{3}}{a t_{F}}$ [12], where $d_{\|}$is the IP component of DMI. Therefore, $\kappa$ can be rewritten as $\kappa=\left(\frac{4}{\pi}\right)^{2} \frac{2 J K_{\text {eff }}}{3 d_{\|}^{2}}$ 
[1] J. Sampaio, V. Cros, S. Rohart, A. Thiaville, and A. Fert, Nucleation, stability and current-induced motion of isolated magnetic skyrmions in nanostructures, Nat. Nanotechnol. 8, 839 (2013).

[2] R. Wiesendanger, Nanoscale magnetic skyrmions in metallic films and multilayers: A new twist for spintronics, Nat. Rev. Mater. 1, 1 (2016).

[3] W. Jiang, X. Zhang, G. Yu, W. Zhang, X. Wang, M. B. Jungfleisch, J. E. Pearson, X. Cheng, O. Heinonen, K. L. Wang, and Y. Zhou, Direct observation of the skyrmion Hall effect, Nat. Phys. 13, 162 (2017).

[4] A. Fert, A. Reyren, and V. Cros, Magnetic skyrmions: Advances in physics and potential applications, Nat. Rev. Mater. 2, 1 (2017).

[5] J. Jena, B. Göbel, V. Kumar, I. Mertig, S. Felser, and S. Parkin, Evolution and competition between chiral spin textures in nanostripes with $D_{2 \mathrm{~d}}$ symmetry, Sci. Adv. 6, eabc0723 (2020).

[6] X. Zhang, Y. Zhou, K. M. Song, T. E. Park, J. Xia, M. Ezawa, X. Liu, W. Zhao, G. Zhao, and S. Woo, Skyrmion-electronics: Writing, deleting, reading and processing magnetic skyrmions toward spintronic applications, J. Phys.: Condens. Matter. 32, 143001 (2020).

[7] K. M. Song, J. S. Jeong, B. Pan, X. Zhang, J. Xia, S. Cha, T. E. Park, K. Kim, S. Finizio, J. Raabe, and J. Chang, Skyrmionbased artificial synapses for neuromorphic computing, Nat. Electron. 3, 148 (2020).

[8] W. Legrand, D. Maccariello, F. Ajejas, S. Collin, A. Vecchiola, K. Bouzehouane, N. Reyren, V. Cros, and A. Fert, Roomtemperature stabilization of antiferromagnetic skyrmions in synthetic antiferromagnets, Nat. Mater. 19, 34 (2020).

[9] I. Dzyaloshinsky, A thermodynamic theory of "weak" ferromagnetism of antiferromagnetics, J. Phys. Chem. Solids 4, 241 (1958).

[10] T. Moriya, Anisotropic superexchange interaction and weak ferromagnetism, Phys. Rev. 120, 91 (1960).

[11] A. Fert and P. M. Levy, Role of Anisotropic Exchange Interactions in Determining the Properties of Spin-Glasses, Phys. Rev. Lett. 44, 1538 (1980).

[12] H. Yang, A. Thiaville, S. Rohart, A. Fert, and M. Chshiev, Anatomy of Dzyaloshinskii-Moriya Interaction at Co/Pt Interfaces, Phys. Rev. Lett. 115, 267210 (2015).

[13] O. Boulle, J. Vogel, H. Yang, S. Pizzini, D. de Souza Chaves, A. Locatelli, T. O. Mentes, A. Sala, L. D. Buda-Prejbeanu, O. Klein, M. Belmeguenai, Y. Roussigné, A. Stashkevich, S. M. Chérif, L. Aballe, M. Foerster, M. Chshiev, F. Auffret, L. M. Miron, and G. Gaudin, Room-temperature chiral magnetic skyrmions in ultrathin magnetic nanostructures, Nat. Nanotechnol. 11, 449 (2016).

[14] A. Soumyanarayanan, M. Raju, A. G. Oyarce, A. K. Tan, M. Y. Im, A. P. Petrović, P. Ho, K. H. Khoo, M. Tran, C. K. Gan, F. Ernult, and C. Panagopoulos, Tunable room-temperature magnetic skyrmions in $\mathrm{Ir} / \mathrm{Fe} / \mathrm{Co} / \mathrm{Pt}$ multilayers, Nat. Mater. 16, 898 (2017).

[15] H. Yang, O. Boulle, V. Cros, A. Fert, and M. Chshiev, Controlling Dzyaloshinskii-Moriya interaction via chirality dependent atomic-layer stacking, insulator capping and electric field, Sci. Rep. 8, 12356 (2018).

[16] M. Raju, A. Yagil, A. Soumyanarayanan, A. K. Tan, A. Almoalem, F. Ma, O. M. Auslaender, and C. Panagopoulos, The evolution of skyrmions in $\mathrm{Ir} / \mathrm{Fe} / \mathrm{Co} / \mathrm{Pt}$ multilayers and their topological Hall signature, Nat. Commun. 10, 1 (2019).
[17] B. Cui, D. Yu, Z. Shao, Y. Liu, H. Wu, P. Nan, Z. Zhu, C. Wu, T. Guo, P. Chen, H. A. Zhou, L. Xi, W. Jiang, H. Wang, S. Liang, H. Du, K. L. Wang, W. Wang, K. Wu, X. Han, G. Zhang, H. Yang, and G. Yu, Néel-type elliptical skyrmions in a laterally asymmetric magnetic multilayer, Adv. Mater. 33, 2006924 (2021).

[18] J. Liang, W. Wang, H. Du, A. Hallal, K. Garcia, M. Chshiev, A. Fert, and H. Yang, Very large Dzyaloshinskii-Moriya interaction in two-dimensional Janus manganese dichalcogenides and its application to realize skyrmion states, Phys. Rev. B 101, 184401 (2020).

[19] Y. Zhang, C. Xu, P. Chen, Y. Nahas, S. Prokhorenko, and L. Bellaiche, Emergence of skyrmionium in a two-dimensional $\mathrm{CrGe}(\mathrm{Se}, \mathrm{Te})_{3}$ Janus monolayer, Phys. Rev. B 102, 241107(R) (2020).

[20] Q. Cui, J. Liang, Z. Shao, P. Cui, and H. Yang, Strain-tunable ferromagnetism and chiral spin textures in two-dimensional Janus chromium dichalcogenides, Phys. Rev. B 102, 094425 (2020).

[21] C. Xu, J. Feng, S. Prokhorenko, Y. Nahas, H. Xiang, and L. Bellaiche, Topological spin texture in Janus monolayers of the chromium trihalides $\mathrm{Cr}(\mathrm{I}, X)_{3}$, Phys. Rev. B 101, 060404(R) (2020).

[22] N. Gao, S. G. Je, M. Y. Im, J. W. Choi, M. Yang, Q. C. Li, T. Y. Wang, S. Lee, H. S. Han, K. S. Lee, W. Chao, C. Hwang, J. Li, and Z. Q. Qiu, Creation and annihilation of topological meron pairs in in-plane magnetized films, Nat. Commun. 10, 1 (2019).

[23] K. W. Moon, J. Yoon, C. Kim, and C. Hwang, Existence of inPlane Magnetic Skyrmion and Its Motion Under Current Flow, Phys. Rev. Appl. 12, 064054 (2019).

[24] B. Göbel, A. Mook, J. Henk, I. Mertig, and O. A. Tretiakov, Magnetic bimerons as skyrmion analogues in in-plane magnets, Phys. Rev. B 99, 060407(R) (2019).

[25] L. Shen, J. Xia, X. Zhang, M. Ezawa, O. A. Tretiakov, X. Liu, G. Zhao, and Y. Zhou, Current-Induced Dynamics and Chaos of Antiferromagnetic Bimerons, Phys. Rev. Lett. 124, 037202 (2020).

[26] X. Li, L. Shen, Y. Bai, J. Wang, X. Zhang, J. Xia, M. Ezawa, O. A. Tretiakov, X. Xu, M. Mruczkiewicz, M. Krawczyk, Y. Xu, R. F. L. Evans, R. W. Chantrell, and Y. Zhou, Bimeron clusters in chiral antiferromagnets, npj Comput. Mater. 6, 1 (2020).

[27] F. Matsukura, Y. Tokura, and H. Ohno, Control of magnetism by electric fields, Nat. Nanotechnol. 10, 209 (2015).

[28] P. J. Hsu, A. Kubetzka, A. Finco, N. Romming, K. von Bergmann, and R. Wiesendanger, Electric-field-driven switching of individual magnetic skyrmions, Nat. Nanotechnol. 12, 123 (2017).

[29] M. Schott, A. Bernand-Mantel, L. Ranno, S. Pizzini, J. Vogel, H. Béa, C. Baraduc, S. Auffret, G. Gaudin, and D. Givord, The skyrmion switch: Turning magnetic skyrmion bubbles on and off with an electric field, Nano Lett. 17, 3006 (2017).

[30] P. Huang, M. Cantoni, A. Kruchkov, J. Rajeswari, A. Magrez, F. Carbone, and H. M. Rønnow, In situ electric field skyrmion creation in magnetoelectric $\mathrm{Cu}_{2} \mathrm{OSeO}_{3}$, Nano Lett. 18, 5167 (2018).

[31] C. Ma, X. Zhang, J. Xia, M. Ezawa, W. Jiang, T. Ono, S. N. Piramanayagam, A. Morisako, Y. Zhou, and X. Liu, Electric field-induced creation and directional motion of domain walls and skyrmion bubbles, Nano Lett. 19, 353 (2018). 
[32] J. Liang, Q. Cui, and H. Yang, Electrically switchable Rashbatype Dzyaloshinskii-Moriya interaction and skyrmion in twodimensional magnetoelectric multiferroics, Phys. Rev. B 102, 220409(R) (2020).

[33] C. Xu, P. Chen, H. Tan, Y. Yang, H. Xiang, and L. Bellaiche, Electric-Field Switching of Magnetic Topological Charge in Type-I Multiferroics, Phys. Rev. Lett. 125, 037203 (2020).

[34] W. Sun, W. Wang, H. Li, G. Zhang, D. Chen, J. Wang, and Z. Cheng, Controlling bimerons as skyrmion analogues by ferroelectric polarization in 2D van der Waals multiferroic heterostructures, Nat. Commun. 11, 1 (2020).

[35] L. Wang, Q. Feng, Y. Kim, R. Kim, K. H. Lee, S. D. Pollard, Y. J. Shin, H. Zhou, W. Peng, D. Lee, W. Meng, H. Yang, J. H. Han, M. Kim, Q. Lu, and T. W. Noh, Ferroelectrically tunable magnetic skyrmions in ultrathin oxide heterostructures, Nat. Mater. 17, 1087 (2018).

[36] G. Kresse and J. Hafner, Ab initio molecular dynamics for liquid metals, Phys. Rev. B 47, 558 (1993).

[37] G. Kresse and J. Furthmuller, Efficient iterative schemes for ab initio total-energy calculations using a plane-wave basis set, Phys. Rev. B 54, 11169 (1996).

[38] G. Kresse and J. Furthmuller, Efficiency of ab-initio total energy calculations for metals and semiconductors using a plane-wave basis set, J. Comput. Mater. Sci. 6, 15 (1996).

[39] Y. Wang and J. P. Perdew, Correlation hole of the spin-polarized electron gas, with exact small-wave-vector and high-density scaling, Phys. Rev. B 44, 13298 (1991).

[40] G. Kresse and D. Joubert, From ultrasoft pseudopotentials to the projector augmented-wave method, Phys. Rev. B 59, 1758 (1999).

[41] J. Li, J. Y. Ni, X. Y. Li, H.-J. Koo, M.-H. Whangbo, J. S. Feng, and H. J. Xiang, Intralayer ferromagnetism between $\mathrm{S}=$ $5 / 2$ ions in $\mathrm{MnBi}_{2} \mathrm{Te}_{4}$ : Role of empty Bi p states, Phys. Rev. B 101, 201408(R) (2020).

[42] Y. Li, Z. Jiang, J. Li, S. Xu, and W. Duan, Magnetic anisotropy of the two-dimensional ferromagnetic insulator $\mathrm{MnBi}_{2} \mathrm{Te}_{4}$, Phys. Rev. B. 100, 134438 (2019).

[43] P. E. Blöchl, Projector augmented-wave method, Phys. Rev. B 50, 17953 (1994).

[44] S. Grimme, J. Antony, S. Ehrlich, and S. Krieg, A consistent and accurate $a b$ initio parametrization of density functional dispersion correction (DFT-D) for the 94 elements H-Pu, J. Chem. Phys. 132, 154104 (2010).

[45] W. Ding, J. Zhu, Z. Wang, Y. Gao, D. Xiao, Y. Gu, Z. Zhang, and W. Zhu, Prediction of intrinsic two-dimensional ferroelectrics in $\mathrm{In}_{2} \mathrm{Se}_{3}$ and other $\mathrm{III}_{2}-\mathrm{VI}_{3}$ van der Waals materials, Nat. Commun. 8, 14956 (2017).

[46] J. Y. You, X. J. Dong, B. Gu, and G. Su, Electric field induced topological phase transition and large enhancements of spin-orbit coupling and Curie temperature in two-dimensional ferromagnetic semiconductors, Phys. Rev. B 103, 104403 (2021).

[47] J. Li, Y. Li, S. Du, Z. Wang, B. L. Gu, S. C. Zhang, K. He, W. Duan, and $\mathrm{Y} . \mathrm{Xu}$, Intrinsic magnetic topological insulators in van der Waals layered $\mathrm{MnBi}_{2} \mathrm{Te}_{4}$-family materials, Sci. Adv. 5, eaaw5685 (2019).

[48] M. M. Otrokov, I. P. Rusinov, M. Blanco-Rey, M. Hoffmann, A. Y. Vyazovskaya, S. V. Eremeev, A. Ernst, P. M. Echenique, A. Arnau, and E. V. Chulkov, Unique ThicknessDependent Properties of the Van Der Waals Interlayer Anti- ferromagnet $\mathrm{MnBi}_{2} \mathrm{Te}_{4}$ Films, Phys. Rev. Lett. 122, 107202 (2019).

[49] S. Yuan, W. F. Io, J. Mao, Y. Chen, X. Luo, and J. Hao, Enhanced piezoelectric response of layered $\mathrm{In}_{2} \mathrm{Se}_{3} / \mathrm{MoS}_{2}$ nanosheet-based van der Waals heterostructures, ACS Appl. Nano Mater. 3, 11979 (2020).

[50] H. Yang, M. Xiao, Y. Cui, L. Pan, K. Zhao, and Z. Wei, Nonvolatile memristor based on heterostructure of 2D roomtemperature ferroelectric $\alpha-\mathrm{In}_{2} \mathrm{Se}_{3}$ and $\mathrm{WSe}_{2}$, Sci. China Inf. Sci. 62, 220404 (2019).

[51] A. Y. Lu, H. Zhu, J. Xiao et al., Janus monolayers of transition metal dichalcogenides, Nat. Nanotechnol. 12, 744 (2017).

[52] R. Sant, M. Gay, A. Marty et al., Synthesis of epitaxial monolayer Janus SPtSe, npj 2D Mater. Appl. 4, 41 (2020).

[53] Y. Zhou, D. Wu, Y. Zhu, Y. Cho, Q. He, X. Yang, K. Herrera, Z. Chu, Y. Han, M. C. Downer, H. Peng, and K. Lai, Out-ofplane piezoelectricity and ferroelectricity in layered $\alpha-\operatorname{In}_{2} \mathrm{Se}_{3}$ nanoflakes, Nano. Lett. 17, 5508 (2017).

[54] The optimized in-plane lattice constants of MBST and $\mathrm{In}_{2} \mathrm{Se}_{3}$ monolayers are 4.233 and $4.108 \AA$, respectively. Here, $1 \times 1$ $\mathrm{In}_{2} \mathrm{Se}_{3}$ monolayer is strained by $3.04 \%$ to match $1 \times 1 \mathrm{MBST}$ monolayer as shown in Fig. 6.

[55] C. Gong, E. Kim, Y. Wang, G. Lee, and X. Zhang, Multiferroicity in atomic van der Waals heterostructures, Nat. Commun. 10, 2657 (2019).

[56] J. Xiao, H. Zhu, Y. Wang, W. Feng, Y. Hu, A. Dasgupta, Y. Han, Y. Wang, D. A. Muller, L. W. Martin, P. Hu, and X. Zhang, Intrinsic Two-Dimensional Ferroelectricity with Dipole Locking, Phys. Rev. Lett. 120, 227601 (2018).

[57] R. F. Evans, W. J. Fan, P. Chureemart, T. A. Ostler, M. O. Ellis, and R. W. Chantrell, Atomistic spin model simulations of magnetic nanomaterials, J. Phys. Condens. Matter 26, 103202 (2014).

[58] S. Z. Lin, A. Saxena, and C. D. Batista, Skyrmion fractionalization and merons in chiral magnets with easy-plane anisotropy, Phys. Rev. B 91, 224407 (2015).

[59] S. Rohart and A. Thiaville, Skyrmion confinement in ultrathin film nanostructures in the presence of Dzyaloshinskii-Moriya interaction, Phys. Rev. B 88, 184422 (2013).

[60] A. O. Leonov, T. I. Monchesky, N. Romming, A. Kubetzka, A. N. Boganov, and R. Wiesendanger, The properties of isolated chiral skyrmions in thin magnetic films, New J. Phys. 18, 065003 (2016).

[61] H. Jia, B. Zimmermann, and S. Blügel, First-principles investigation of chiral magnetic properties in multilayers: $\mathrm{Rh} / \mathrm{Co} / \mathrm{Pt}$ and $\mathrm{Pd} / \mathrm{Co} / \mathrm{Pt}$, Phys. Rev. B 98, 144427 (2018).

[62] D. -S. Wang, R. Wu, and A. J. Freeman, First-principles theory of surface magnetocrystalline anisotropy and the diatomic-pair model, Phys. Rev. B 47, 14932 (1993).

[63] Q. Cui, J. Liang, B. Yang, Z. Wang, P. Li, P. Cui, and H. Yang, Giant enhancement of perpendicular magnetic anisotropy and induced quantum anomalous Hall effect in graphene/ $\mathrm{NiI}_{2}$ heterostructures via tuning the van der Waals interlayer distance, Phys. Rev. B 101, 214439 (2020).

[64] B. Yang, X. Zhang, H. Yang, X. Han, and Y. Yan, Nonmetallic atoms induced magnetic anisotropy in monolayer chromium trihalides, J. Phys. Chem. C 123, 691 (2019). 
[65] R. F. W. Bader, Atoms in Molecules - A Quantum Theory (Oxford University Press, Oxford, 1990).

[66] G. Henkelman, A. Arnaldsson, and H. Jónsson, A fast and robust algorithm for Bader decomposition of charge density, Comput. Mater. Sci. 36, 354 (2006).

[67] E. Sanville, S. D. Kenny, R. Smith, and G. Henkelman, Improved grid-based algorithm for Bader charge allocation, J. Comput. Chem. 28, 899 (2007).

[68] W. Tang, E. Sanville, and G. Henkelman, A grid-based Bader analysis algorithm without lattice bias, J. Phys. Condens. Matter 21, 084204 (2009).

[69] F. Xue, Z. Wang, Y. Hou, L. Gu, and R. Wu, Control of magnetic properties of $\mathrm{MnBi}_{2} \mathrm{Te}_{4}$ using a van der Waals ferroelectric $\mathrm{III}_{2}-\mathrm{VI}_{3}$ film and biaxial strain, Phys. Rev. B 101, 184426 (2020).
[70] A. Togo and I. Tanaka, First principles phonon calculations in materials science, Scr. Mater. 108, 1 (2015).

[71] S. Nosé, A unified formulation of the constant temperature molecular dynamics methods, J. Chem. Phys. 81, 511 (1984).

[72] G. H. O. Daalderop, P. J. Kelly, and M. F. H. Schuurmans, Firstprinciples calculation of the magnetocrystalline anisotropy energy of iron, cobalt, and nickel, Phys. Rev. B 41, 11919 (1990).

[73] A. Hallal, H. Yang, B. Dieny, and M. Chshiev, Anatomy of perpendicular magnetic anisotropy in $\mathrm{Fe} / \mathrm{MgO}$ magnetic tunnel junctions: First-principles insight, Phys. Rev. B 88, 184423 (2013).

[74] Y. S. Hou and R. Q. Wu, Strongly Enhanced Gilbert Damping in 3d Transition-Metal Ferromagnet Monolayers in Contact with the Topological Insulator $\mathrm{Bi}_{2} \mathrm{Se}_{3}$, Phys. Rev. Appl. 11, 054032 (2019). 